\title{
BMJ Open Exercise interventions for ankylosing spondylitis: a protocol for a Bayesian network meta-analysis
}

\author{
Shun-Li Kan, ${ }^{1}$ Ling-Xiao Chen, ${ }^{\circ} 2$ Zhi-Fang Yuan, ${ }^{3}$ Wei Hu, ${ }^{1}$ Ru-Sen Zhu ${ }^{1}$
}

\begin{abstract}
To cite: Kan S-L, Chen L-X, Yuan Z-F, et al. Exercise interventions for ankylosing spondylitis: a protocol for a Bayesian network meta-analysis. BMJ Open 2019;9:e029991. doi:10.1136/ bmjopen-2019-029991

- Prepublication history and additional material for this paper are available online. To view these files, please visit the journal online (http://dx.doi. org/10.1136/bmjopen-2019029991).
\end{abstract}

Received 21 February 2019 Revised 17 May 2019 Accepted 22 May 2019
Check for updates

(C) Author(s) (or their employer(s)) 2019. Re-use permitted under CC BY-NC. No commercial re-use. See rights and permissions. Published by BMJ.

${ }^{1}$ Department of Spine Surgery, Tianjin Union Medical Center, Tianjin, China

${ }^{2}$ Institute of Bone and Joint Research, The Kolling Institute, Sydney Medical School, University of Sydney, Sydney, New South Wales, Australia ${ }^{3}$ Department of Respiratory and Critical Care Medicine, Tianjin Medical University General Hospital, Tianjin, China

Correspondence to

Dr Ru-Sen Zhu;

rszhuspine@163.com

\begin{abstract}
Introduction Ankylosing spondylitis (AS) is a universal chronic inflammatory rheumatic disease which predominantly results in chronic back pain and stiffness. However, some patients suffering from AS do not react well to pharmacological interventions. Exercise intervention has been employed for the treatment of AS and works as a complementary part of the management of AS. However, the effect of different types of exercise interventions remains unclear. The purpose of this study is to determine the relative efficacy of different types of exercise interventions for individuals with AS using a Bayesian network meta-analysis.

Methods and analysis We will conduct a systematic literature review of randomised controlled trials that compare different types of exercise interventions for individuals with AS. PubMed, EMBASE and the Cochrane Library will be searched up to February 2019. The primary outcomes are functional capacity, pain and disease activity. The risk of bias for individual studies will be evaluated according to the Cochrane Handbook. A Bayesian network meta-analysis will be performed to compare the efficacy of different types of exercise interventions. The quality of evidence will be assessed by the Grading of Recommendations, Assessment, Development and Evaluation approach.

Ethics and dissemination Ethical approval and patient consent are not required as this study is a meta-analysis based on published studies. The results of this network meta-analysis will be submitted to a peer-reviewed journal for publication.
\end{abstract}

PROSPERO registration number CRD42019123099.

\section{INTRODUCTION}

Ankylosing spondylitis (AS) is a universal chronic inflammatory rheumatic disease which predominantly influences the axial skeleton (eg, spine, hips and shoulders).${ }^{12} \mathrm{AS}$ is characterised by inflammatory back pain which is caused by sacroiliitis and spondylitis. ${ }^{1}$ Inflammatory back pain may happen in $70 \%-80 \%$ of patients with AS. AS commonly starts early and about $10 \%-20 \%$ of patients with AS commence to develop the first symptoms before 16 years of age. ${ }^{34}$ It has been reported that estimates for the prevalence of AS vary from $0.01 \%$ to $1.8 \% .{ }^{5}$ Patients

\section{Strengths and limitations of this study}

This is the most comprehensive review comparing the efficacy of different types of exercise interventions for individuals with ankylosing spondylitis through a Bayesian network meta-analysis.

- The main strength is that only randomised controlled trials will be included.

- We will use the Grading of Recommendations Assessment, Development and Evaluation approach to evaluate the quality of evidence.

- The duration of some trials is too short to provide decisive evidence on the effects of exercise interventions.

with AS often experience chronic back pain, stiffness, arthritis and enthesitis, which seriously affect patients' health and quality of life, disturb their recreational activities, work, family life and relationships, and result in considerable psychological distress and fears.

Non-steroidal anti-inflammatory drugs (NSAIDs), including COX-2 inhibitors, are recommended as the first-line drug intervention for reducing pain and stiffness. Biological disease-modifying antirheumatic drugs have also proved effective to manage inhibitors, the anti-interleukin- 17 inhibitor and so on. ${ }^{6}$ However, some patients suffering from AS do not react well to pharmacological interventions. ${ }^{7}$ Exercise is recommended by several guidelines as a co-intervention in combination with pharmacological interventions to treat patients with AS. ${ }^{28}$ Previous systematic reviews ${ }^{9-11}$ demonstrated that exercises have significant positive effects on pain, spinal mobility and physical function. However, they did not classify different types of exercise, such as group exercise, individualised exercise, supervised exercise, home-based exercise and so on. Therefore, we do not know which is the best one. When no studies exist that directly compare all relevant treatment choices, a network meta-analysis can be performed by comparing the relative effects of treatments 
against a common comparator or combining a variety of comparisons that are taken together from one or more chains linking the treatments of interest. ${ }^{12}$

Therefore, the purpose of this study is to comprehensively review the literature and determine the relative efficacy of different types of exercise interventions for individuals with AS using a Bayesian network meta-analysis.

\section{METHODS \\ Design}

A network meta-analysis using a Bayesian framework will be implemented in this study. This protocol of network meta-analysis will be performed on the basis of the Preferred Reporting Items for Systematic review and Meta-Analysis Protocol (PRISMA-P), ${ }^{13}$ and the reporting of the following network meta-analysis will obey the PRISMA extension statement for reporting of systematic reviews incorporating network meta-analysis of healthcare interventions. ${ }^{14}$ This study has been registered at PROSPERO (http://www.crd.york.ac.uk/PROSPERO) with registration number CRD42019123099.

\section{Eligibility criteria}

Type of study

We will include randomised controlled trials comparing different exercise interventions, and/or comparing a specific exercise intervention with no treatment, standard care or usual physical activity. For cross-over studies, we only use the data before the wash-out period. We will not restrain the language or date of publication. We will divide the trial duration into a short-term follow-up (6 months) and long-term follow-up (12 months). If the trial duration is closer to 6 or 12 months, we will classify the trial duration as a short-term follow-up or long-term follow-up.

\section{Participants}

Trials enrolling adults, aged at least 18 years, with a diagnosis of AS according to the Modified New York criteria ${ }^{15}$ or the Amor criteria ${ }^{16}$ or radiographic axial spondyloarthritis (SpA) according to the criteria for axial SpA defined by the Assessment of Spondyloarthritis International Society (ASAS) ${ }^{17}$ will be included.

We will exclude studies involving participants with non-radiographic axial SpA according to the criteria for axial SpA defined by the ASAS.

\section{Type of interventions}

Any type of exercise interventions will be included. Exercise intervention is defined as a type of physical activity that is planned, structured and repeated over a period of time. $^{18}$

Trials that compare an exercise intervention combined with a co-intervention versus the co-intervention alone or the exercise intervention alone (eg, an exercise intervention plus anti-tumour necrosis factor (TNF) $\alpha$ therapy vs anti-TNF $\alpha$ therapy alone, an exercise intervention plus spa therapy vs the exercise intervention) will be considered.

Trails investigating exercise interventions with a different setting (home, hospital or elsewhere) or different delivery method (individual, group, supervision or mixed) will be included.

Trials comparing an exercise intervention with no treatment, standard care or usual physical activity will be considered.

\section{Outcomes of interest \\ Primary outcomes}

The Bath Ankylosing Spondylitis Functional Index $(\mathrm{BASFI})^{19}$ is a 10 -item index that evaluate the functional capacity in performing daily activities of patients with AS. Higher score of the BASFI reflects greater impairment in functional capacity.

The pain will be measured based on a visual analogue scale or numerical rating scale. We will record data on back pain at night, total back pain, overall pain at night or overall pain. We will collect the highest pain score from the mentioned alternatives. And the highest pain score on numeric value will be regarded as the final pain score.

The Bath Ankylosing Spondylitis Disease Activity Index $(\mathrm{BASDAI})^{20}$ is the gold standard for measuring and evaluating disease activity in AS. Higher score of the BASDAI indicates greater disease activity.

\section{Secondary outcomes}

The Medical Outcomes Study 36-Item Short-Form General Health Survey (SF-36) will be used to evaluate the quality of life, with higher scores indicating better quality of life.

The Bath Ankylosing Spondylitis Metrology Index $(\mathrm{BASMI})^{21}$ is the most widely reported, validated objective axial mobility measure, which consists of five steps: cervical rotation, tragus to wall distance, lumbar side flexion, modified Schober's test and intermalleolar distance. High scores mean severer limitations of movement.

\section{Data sources and search strategy}

We will systematically search PubMed, EMBASE and the Cochrane Library for primary studies up to February 2019. The search strategy will combine free text words and medical subject headings regarding exercise, spondyloarthritis and randomised controlled trials. The detail of the search strategy for PubMed is shown in the online supplementary file S1. This search strategy will be modified as required for other databases. Furthermore, we will also retrieve the WHO International Clinical Trials Registry Platform and ClinicalTrials.gov to identify ongoing trial registers. We will examine the bibliographies of pertinent systematic reviews and meta-analyses for additional related studies. We will not limit the language of publication or publication period.

\section{Study selection}

Two reviewers will independently check the titles and abstracts through the initial retrieval. Publications not 
fulfilling the eligibility criteria will be eliminated. After excluding the irrelevant publications, we will examine the full text of the remaining publications based on the same eligibility criteria. Any discrepancies will be settled by discussion and consensus. Excluded publications and the reasons for exclusion will be reported and confirmed by a third investigator.

\section{Data extraction}

Data from included publications will be independently extracted by two reviewers using a standardised data abstraction list. The following characteristic information will be extracted: study characteristics (first author, publication year, study year, number of centres, country and sponsor), patient characteristics (sample size, mean age, gender ratio, the stage of the disease and inclusion/ exclusion criteria), intervention details for each treatment group (eg, number of intervention groups, exercise modality and the detailed description, frequency and duration of the intervention, the duration of follow-up and co-interventions) and outcome measures (BASFI, BASDAI, BASMI, pain and SF-36). We will prioritise the data at the end of the studies compared with the changes from baseline in all the outcomes. Numerical data will be extracted to calculate pooled estimations. If the study only reports $\mathrm{SE}$, $\mathrm{p}$ value or $\mathrm{CI}$, we will convert them into $\mathrm{SD}{ }^{22}$ If the study reports median and IQR, we will calculate SD by dividing the IQR by 1.35 and considering the median equivalent to the mean. ${ }^{22}$ If the data are not reported in the texts directly, we will infer them from the associated graphs. If data cannot be obtained, we will contact the corresponding authors. Any disagreements will be settled by discussion and consensus.

\section{Risk of bias assessment}

The Cochrane Risk of Bias Tool will be used to appraise the risk of bias for individual studies. ${ }^{23}$ Each study will be evaluated and scored as high, low or unclear risk of bias based on the following criteria: randomisation, allocation concealment, blinding of participants and personnel, blinding of outcome assessors, incomplete outcome data, selective reporting and other biases. A study with a high risk of bias in one or more domains will be viewed as high risk of bias. A study with a low risk of bias in all domains will be considered as low risk of bias. If not, a study will be treated as unclear risk of bias. Any disagreements were resolved by discussion and consensus.

\section{Statistical analysis}

A traditional pairwise meta-analysis will be done when at least two studies exist for an outcome. A random-effects model with the Hartung-Knapp-Sidik-Jonkstra method $^{24}$ will be used to estimate the effect size and $95 \%$ CI accounting for methodological and clinical heterogeneity across studies, with Stata V.13.0. ${ }^{25}$ We will use mean difference (MD) for a certain outcome when $>50 \%$ studies reporting the outcome use the same measurement. Otherwise, standardised MD will be used. The extent of between-trial heterogeneity will be assessed with $\mathrm{I}^{2}$ statistic, with values over $50 \%$ indicating considerable heterogeneity. ${ }^{26}$

We will perform network meta-analyses to merge direct and indirect comparisons. All network meta-analyses will be conducted using a Bayesian Markov chain Monte Carlo (MCMC) framework in R V.3.2.5 software (https:// cran.r-project.org/src/base/R-3/) via the gemtc V.0.8-2 package. MD and $95 \%$ credible interval will be used as summary statistics to quantify the effect of different exercise interventions. Random-effects and consistency models will be adopted in this network meta-analysis, as they are considered to be the most conservative approach to dealing with between-study heterogeneity. ${ }^{27}$ To generate posterior distributions of model parameters, 150000 iterations of MCMC after 50000 tuning iterations in three chains will be run. ${ }^{28}$ The convergence of iterations will be examined with the Gelman-Rubin-Brooks diagnostic plots. ${ }^{29}$ For any specific outcome, we will rank the probability of each intervention being the best (superior to all other interventions), second best, third best and so on.

The posterior mean residual deviance, an absolute measure of fit, will be computed. The value of posterior mean residual deviance and the number of independent data points will be assessed to check if the model fits the data satisfactorily. ${ }^{30}$ To appraise the consistency, we will use the following methods. First, the model fit from the consistency model will be compared with that from the inconsistency model. ${ }^{31}$ Second, network meta-analysis results (indirect evidence using the node-split approach) will be compared with pairwise meta-analysis results (direct evidence in a frequentist framework). ${ }^{32}$

Clinical and methodological heterogeneity will be evaluated by checking the characteristics and design of the included studies. Statistical heterogeneity in the network will be assessed according to the heterogeneity parameter $\left(\mathrm{I}^{2}\right.$ or $\tau^{2}$ derived from the network meta-analysis. $\mathrm{I}^{2}>50 \%$ indicates substantial heterogeneity. Heterogeneity will be explored by fitting covariates (ie, mean age, sample size, the duration of symptoms, the dose of exercise (frequency $x$ duration intensity) and the duration of follow-up) in network meta-regression analyses. ${ }^{33}$ Subgroup analyses will be further conducted ground on the duration of symptoms (early or long-term disease) and concomitant pharmacological treatment (anti-TNF agents, NSAIDs or other pharmacological interventions), if possible. Sensitivity analyses will be executed to test the robustness of outcomes by limiting analyses to studies with low risk of bias.

To examine the potential of small-study effects in the network, comparison-adjusted funnel plots will be produced. ${ }^{34}$ For the comparison-adjusted funnel plot, the horizontal axis will represent the difference between study-specific effect sizes and the comparison-specific summary effect. In the absence of small-study effects, the comparison-adjusted funnel plot should be symmetric around the zero line. 


\section{Quality of evidence}

We will follow the Grading of Recommendations, Assessment, Development and Evaluation four-step approach to grade the quality of treatment effect estimations from network meta-analysis. ${ }^{35}$ First, present direct and indirect treatment estimates for each comparison of the evidence network. Second, rate the quality of each direct and indirect effect estimate. Then, present the network meta-analysis estimate for each comparison of the evidence network. At last, rate the quality of each network meta-analysis effect estimate. According to the risk of bias, inconsistency, indirectness, imprecision and publication bias, the quality of evidence will be graded as high, moderate, low or very low.

\section{Patient and public involvement \\ Patients or the public will not be involved.}

\section{ETHICS AND DISSEMINATION \\ Ethical issues}

As no primary data collection will be undertaken, no additional formal ethical assessment and no informed consent are required.

\section{Publication plan}

This network meta-analysis will be submitted to a peer-reviewed journal. It will be disseminated electronically and in print.

Contributors S-LK, L-XC, Z-FY and R-SZ: participated in the conception and design of the study, including search strategy development. S-LK, L-XC, Z-FY and WH: tested the feasibility of the study. S-LK: wrote the manuscript. All the authors critically reviewed this manuscript and approved the final version.

Funding This work was supported by Tianjin Municipal Science and Technology Commission (16KG158), and Foundation of Tianjin Union Medical Center (2018YJ010). The funders had no role in study design, data collection and analysis, decision to publish, or preparation of the manuscript.

Competing interests None declared.

Provenance and peer review Not commissioned; externally peer reviewed.

Open access This is an open access article distributed in accordance with the Creative Commons Attribution Non Commercial (CC BY-NC 4.0) license, which permits others to distribute, remix, adapt, build upon this work non-commercially, and license their derivative works on different terms, provided the original work is properly cited, appropriate credit is given, any changes made indicated, and the use is non-commercial. See: http://creativecommons.org/licenses/by-nc/4.0/.

\section{REFERENCES}

1. Baraliakos X, Haibel H, Listing J, et al. Continuous long-term antiTNF therapy does not lead to an increase in the rate of new bone formation over 8 years in patients with ankylosing spondylitis. Ann Rheum Dis 2014;73:710-5.

2. van der Heijde D, Ramiro S, Landewé R, et al. 2016 update of the ASAS-EULAR management recommendations for axial spondyloarthritis. Ann Rheum Dis 2017;76:978-91.

3. Colbert RA. Classification of juvenile spondyloarthritis: Enthesitisrelated arthritis and beyond. Nat Rev Rheumatol 2010;6:477-85.

4. Ramanathan A, Srinivasalu H, Colbert RA. Update on juvenile spondyloarthritis. Rheum Dis Clin North Am 2013;39:767-88.

5. van Tubergen $A$. The changing clinical picture and epidemiology of spondyloarthritis. Nat Rev Rheumatol 2015;11:110-8.

6. Taurog JD, Chhabra A, Colbert RA. Ankylosing Spondylitis and Axial Spondyloarthritis. N Engl J Med 2016;374:2563-74.
7. Sieper J, Braun J, Dougados M, et al. Axial spondyloarthritis. Nat Rev Dis Primers 2015;1:15013.

8. Ward MM, Deodhar A, Akl EA, et al. American College of Rheumatology/Spondylitis Association of America/Spondyloarthritis Research and Treatment Network 2015 Recommendations for the Treatment of Ankylosing Spondylitis and Nonradiographic Axial Spondyloarthritis. Arthritis Rheumatol 2016;68:282-98.

9. Dagfinrud H, Hagen KB, Kvien TK. Cochrane Musculoskeletal Group. Physiotherapy interventions for ankylosing spondylitis. Cochrane Database Syst Rev 2008;35:Cd002822.

10. Martins NA, Furtado GE, Campos MJ, et al. Exercise and ankylosing spondylitis with New York modified criteria: a systematic review of controlled trials with meta-analysis. Acta Reumatol Port 2014;39:298-308.

11. Pécourneau V, Degboé $Y$, Barnetche $T$, et al. Effectiveness of Exercise Programs in Ankylosing Spondylitis: A Meta-Analysis of Randomized Controlled Trials. Arch Phys Med Rehabil 2018;99:383-9.

12. Lu G, Ades AE. Combination of direct and indirect evidence in mixed treatment comparisons. Stat Med 2004;23:3105-24.

13. Moher D, Shamseer L, Clarke M, et al. Preferred reporting items for systematic review and meta-analysis protocols (PRISMA-P) 2015 statement. Syst Rev 2015;4:1.

14. Hutton B, Salanti G, Caldwell DM, et al. The PRISMA extension statement for reporting of systematic reviews incorporating network meta-analyses of health care interventions: checklist and explanations. Ann Intern Med 2015;162:777-84.

15. van der Linden S, Valkenburg HA, Cats A. Evaluation of diagnostic criteria for ankylosing spondylitis. A proposal for modification of the New York criteria. Arthritis Rheum 1984;27:361-8.

16. Amor B, Dougados M, Mijiyawa M. [Criteria of the classification of spondylarthropathies]. Rev Rhum Mal Osteoartic 1990;57:85-9.

17. Rudwaleit $M$, van der Heijde $D$, Landewé $R$, et al. The development of Assessment of SpondyloArthritis international Society classification criteria for axial spondyloarthritis (part II): validation and final selection. Ann Rheum Dis 2009;68:777-83.

18. Bouchard C, Blair S, Haskell W. Physical Activity and Health. Human Kinetics, Inc 2007.

19. Calin A, Garrett S, Whitelock H, et al. A new approach to defining functional ability in ankylosing spondylitis: the development of the Bath Ankylosing Spondylitis Functional Index. J Rheumatol 1994;21:2281-5.

20. Garrett S, Jenkinson T, Kennedy LG, et al. A new approach to defining disease status in ankylosing spondylitis: the Bath Ankylosing Spondylitis Disease Activity Index. J Rheumatol 1994;21:2286-91.

21. Jenkinson TR, Mallorie PA, Whitelock HC, et al. Defining spinal mobility in ankylosing spondylitis (AS). The Bath AS Metrology Index. $J$ Rheumatol 1994;21:1694-8.

22. Higgins JPT, Green S, eds. Cochrane Handbook for Systematic Reviews of Interventions Version 5.1.0 : The Cochrane Collaboration, 2011.

23. Higgins JP, Altman DG, Gøtzsche PC, et al. The Cochrane Collaboration's tool for assessing risk of bias in randomised trials. BMJ 2011;343:d5928.

24. Veroniki AA, Jackson D, Viechtbauer W, et al. Recommendations for quantifying the uncertainty in the summary intervention effect and estimating the between-study heterogeneity variance in randomeffects meta-analysis. The Cochrane database of systematic reviews 2015:25-7.

25. DerSimonian R, Laird N. Meta-analysis in clinical trials. Control Clin Trials 1986;7:177-88.

26. Higgins JP, Thompson SG, Deeks JJ, et al. Measuring inconsistency in meta-analyses. BMJ 2003;327:557-60.

27. Mills EJ, Thorlund K, loannidis JP. Demystifying trial networks and network meta-analysis. BMJ 2013;346:f2914.

28. Toft N, Innocent GT, Gettinby G, et al. Assessing the convergence of Markov Chain Monte Carlo methods: an example from evaluation of diagnostic tests in absence of a gold standard. Prev Vet Med 2007;79(2-4):244-56.

29. Gelman A, Rubin DB. Markov chain Monte Carlo methods in biostatistics. Stat Methods Med Res 1996;5:339-55.

30. Dias S, Sutton AJ, Ades AE, et al. Evidence synthesis for decision making 2: a generalized linear modeling framework for pairwise and network meta-analysis of randomized controlled trials. Med Decis Making 2013;33:607-17.

31. Dias S, Welton NJ, Sutton AJ, et al. Evidence synthesis for decision making 4: inconsistency in networks of evidence based on randomized controlled trials. Med Decis Making 2013;33:641-56.

32. Dias S, Welton NJ, Caldwell DM, et al. Checking consistency in mixed treatment comparison meta-analysis. Stat Med 2010;29(78):932-44. 
33. Dias S, Sutton AJ, Welton NJ, et al. Evidence synthesis for decision making 3: heterogeneity--subgroups, meta-regression, bias, and bias-adjustment. Med Decis Making 2013;33:618-40.

34. Chaimani A, Higgins JP, Mavridis D, et al. Graphical tools for network meta-analysis in STATA. PLoS One 2013;8:e76654.
35. Puhan MA, Schünemann HJ, Murad MH, et al. A GRADE Working Group approach for rating the quality of treatment effect estimates from network meta-analysis. BMJ 2014;349:g5630. 